\title{
Change in the Membranous Lipid Composition Accelerates Lipid Peroxidation in Young Rat Hearts Subjected to 2 Weeks of Hypoxia Followed by Hyperoxia
}

\author{
Tatsujiro Oka, MD; Toshiyuki Itoi, MD; Naoto Terada, MD*; Hiroki Nakanishi, PhD**; \\ Ryo Taguchi, PhD**; Kenji Hamaoka, MD
}

\begin{abstract}
Background The effects of chronic hypoxia on cardiac membrane fatty acids and on lipid peroxidation were examined, as well as the effect of l-carnitine (LCAR), which suppresses lipid peroxidation, on this process. Methods and Results Four-week-old Sprague-Dawley rats were exposed to 10\% oxygen for 14 days ("Hypoxia"), and then to $100 \%$ oxygen for $12 \mathrm{~h}\left(\mathrm{O}_{2}\right)$. LCAR $(200 \mathrm{mg} / \mathrm{kg})$ was administered by intraperitoneal injection daily for 2 weeks. Fatty acid composition, malondialdehyde (MDA) as a lipid peroxidation product, and antioxidants (superoxide dismutase (SOD), glutathione peroxidase and catalase) were measured. The concentration of linoleic acid was lower, and that of docosahexaenoic acid, which has more double bonds than linoleic acid, was increased in hypoxic hearts. SOD activity decreased in hypoxia, whereas MDA was unchanged, but significantly increased in "Hypoxia"+O2. LCAR reduced the increase in MDA, and had no effect on SOD activity or fatty acid composition. The administration of LCAR caused an increase in the ventricular levels of acetylcarnitine.

Conclusions These results suggest that chronic hypoxia changes the cardiac fatty acid composition of juvenile rats to fatty acids that contain more double-bonds and reduce SOD activity, and that lipid peroxidation was augmented by exposure to oxygen. (Circ J 2008; 72: 1359-1366)
\end{abstract}

Key Words: Chronic hypoxia; Fatty acid; L-carnitine; Lipid peroxidation

$\mathbf{P}$ rogress in pediatric cardiac surgery in the past few decades has made a tremendous impact on outcomes. The mortality rate following complex defect repair has decreased. However, the association of cyanosis with congenital heart disease (CHD) is responsible for worse postoperative ventricular function and a more complex postoperative hospital course 1,2 Hypoxia causes complex physiologic responses, in other words, angiogenesis, and the production of erythropoietin, and is associated with an increased risk of stroke and thromboembolism.

Free radicals cause increased lipid peroxidation during myocardial ischemia and reperfusion, and they are important mediators of cellular damage,, 6 However, the mechanism of increased lipid peroxidation in response to chronic hypoxia has not been adequately studied. Furthermore, there is little detailed information about the composition of lipid bilayers, which are mainly composed of phospholipids, under hypoxic conditions. Because membrane fatty acids with more than 2 double bonds tend to be more readily damaged by free radicals, it is likely that lipid peroxide

(Received March 9, 2007; revised manuscript received February 6, 2008; accepted March 19, 2008)

Department of Pediatric Cardiology and Nephrology, *Department of Pediatrics, Graduate School of Medical Science, Kyoto Prefectural University of Medicine, Kyoto and **Department of Metabolome, Graduate School of Medicine, The University of Tokyo, Tokyo, Japan Mailing address: Toshiyuki Itoi, MD, Department of Pediatric Cardiology and Nephrology, Graduate School of Medical Science, Kyoto Prefectural University of Medicine, 465 Kajii-cho, Kawaramachi, Hirokoji, Kamigyo-ku, Kyoto 602-8566, Japan. E-mail: titoi@koto. kpu-m.ac.jp

All rights are reserved to the Japanese Circulation Society. For permissions, please e-mail: cj@j-circ.or.jp production might be increased in membranes with higher amounts of unsaturated fatty acids.

Acute myocardial injury in pediatric cardiovascular surgery is a major problem that is not found to the same extent in adult cardiovascular surgery, 2,5 possibly because the free radical scavenging system of the myocardium in patients with cyanotic CHD may not neutralize the superoxide ion radicals that are formed by a sudden increase in arterial oxygen tension or by reperfusion after ischemic arrest.,5,7 Oxygen-mediated reperfusion injury is caused by the production of oxygen free radicals generated by the rapid reintroduction of oxygen?

Although previous experimental studies have shown that initiating either cardiopulmonary bypass at a lower fraction of inspired oxygen ${ }^{8}$ or leukocyte filtration may decrease free radical injury5, effective pharmacotherapy to prevent the acceleration of lipid peroxidation during the ischemic reperfusion stage of pediatric cardiovascular surgery has not been sufficiently examined. Carnitine is a vital component in lipid metabolism for the production of adenosine triphosphate through $\beta$-oxidation and subsequent oxidative phosphorylation. L-carnitine (LCAR) can suppress lipid peroxidation after ischemia reperfusion; ${ }^{9-11}$ however, there have been few studies showing the effect of LCAR in cyanotic CHD models, and no study has examined the effects of LCAR therapy on cardiac fatty acid composition.

The purpose of this study was to evaluate fatty acid composition and lipid peroxidation in the immature hypoxic myocardium, and to determine the effect of hypoxia on the myocardial antioxidant system. The present model is a close relation to cyanotic CHD after repair. As previous studies have demonstrated that hypoxic alterations reach a level of steady-state by 2 weeks, 2 we believe this time period of 
hypoxia can be used to generate an experimental model that effectively reflects cyanotic CHD. An additional goal was to elucidate the effect of LCAR on the composition of fatty acids, antioxidants, and the concentration of carnitine in the hypoxic myocardium.

\section{Methods}

\section{Animal Preparation and Treatment}

Four-week-old male Sprague-Dawley rats were purchased from Shimizu laboratory supplies (Kyoto, Japan). The total number of rats required for the various groups used in this study was 75 . The rats were exposed to $10 \%$ oxygen in a mixture of nitrogen and room air in a chamber for 2 weeks ("Hypoxia") ( $n=28) ! 3$ Half of them were then exposed to $100 \%$ oxygen for $12 \mathrm{~h}\left(+\mathrm{O}_{2}\right)$. The $\mathrm{O}_{2}$ concentration in the chamber was monitored by an analyzer. Five rats were reared in each condition using a 12-h light-dark cycle. The chamber was opened twice a week for a few minutes to clean the cages. Control age-matched normoxic rats were maintained in room air for the same time period ("Normoxia") $(n=14)$. Standard rat chow (CRF-1; Charles River, Yokohama, Japan) and water were provided ad libitum. LCAR $(200 \mathrm{mg} / \mathrm{kg})$ was administered by intraperitoneal injection daily for 2 weeks to a group of rats in the hypoxic chamber (+LCAR) ( $n=7$ per group). During the injection, the mouth of the rat was covered with a mask that delivered $10 \%$ oxygen. Vehicle $(0.9 \% \mathrm{NaCl})$ was injected into the rats by intraperitoneal injection daily $(n=5)$.

At the time of death, the rats were sedated with sodium pentobarbital $(50 \mathrm{mg} / \mathrm{kg})$, intubated, and ventilated automatically with $10 \%$ oxygen. The heart was removed into normoxic-cooled $\left(4^{\circ} \mathrm{C}\right)$ saline; the ascending aorta was cannulated and the heart was flushed with ice-cold saline to remove any blood in the coronary arteries. The heart was dissected from other tissue and weighed. It is well known that chronic hypoxia alters the pulmonary hemodynamics and leads to pulmonary hypertension, with selective hypertrophy of the right ventricle. We analyzed the left ventricle to exclude the influence of pulmonary hypertension. The heart weight was assessed by measurement of the weight of the right ventricular free wall and septum plus the weight of the left ventricular (LV) free wall. The LVs were frozen in liquid nitrogen and stored at $-80^{\circ} \mathrm{C}$ until analysis.

The protocol was reviewed by the Committee of Ethics on Animal Experiments, Kyoto Prefectural University Graduate School of Medicine, and was carried out in accordance with the Guidelines for Animal Experiments, Kyoto Prefectural University of Medicine. The investigation conformed to the Guide for the Care and Use of Laboratory Animals published by the US National Institutes of Health (NIH Publication No. 85-23, revised 1996).

\section{Quantitative Fatty Acid Analysis}

Total fatty acids were extracted from tissue samples according to the Bligh-Dyer method ${ }^{14}$ In brief, the LV tissue was extracted with methanol/chloroform/water (2:1:0.8 by volume). After centrifugation, a biphasic system was produced by dilution of the extract with chloroform and saline solution (1:1 by vol.). The lower layer was withdrawn. Individual phospholipid classes were separated by thin layer chromatography. 15 The fatty acid and phospholipid fractions were methylated using $5 \%$ hydrogen chloride-methanol at $100^{\circ} \mathrm{C}$ for $1 \mathrm{~h}$. Butylated hydroxy toluene $(0.05 \mathrm{~g} / \mathrm{L})$ was added to the methanol to prevent peroxidation of the fatty acids during methylation. Heptadecanoic acid was added to the lipid extract as an internal standard. The fatty acid methyl esters were then analyzed using a Shimadzu gas chromatograph (QP5000) and mass spectrometer (GC17A), equipped with a $30 \mathrm{~m} \times 0.25 \mathrm{~mm} \mathrm{DB}-23$ column that was coated with (50\% cyanopropyl)-methylpolysiloxane (J\&W Scientific, Folsom, CA, USA).

Electrospray ionization mass spectrometry (ESI-MS) was used to elucidate the structure of the phospholipids. The lipids were extracted from the rat ventricles by BlighDyer's method 13 The ESI-MS analysis was performed using a 4000Q TRAP quadropole-linear ion trap hybrid mass spectrometer (Applied Biosystems/MDS Sciex, Concord, ON, Canada) with a LC-10AD VPu HPLC system combined with a SIL-10AD VP autosampler (Shimadzu, Kyoto, Japan).

The conditions used for the 4000Q TRAP were as follows. The mobile phase composition was acetonitrile/methanol/ water $(6: 7: 2)$ containing $0.1 \%$ ammonium formate $(\mathrm{pH} 7.4)$. The flow rate was $8 \mu 1 / \mathrm{min}$. The collision energy used varied according to the desired experiment. Typically, $5 \mu 1$ of the sample was used. Mass spectra of phosphatidylethanolamines (PEs) were detected by a fatty-acyl survey. The molecules including 16:0 and 18:0 were detected by precursor ion scanning of $\mathrm{m} / \mathrm{z} 255.2$ and $\mathrm{m} / \mathrm{z} 283.2$ in the negative ion mode.

\section{Biochemical Assays}

The determination of thiobarbituric acid reactive-substances (TBARs) in the heart was performed according to a modified method of Ohkawa 16 The absorbance was measured at $532 \mathrm{~nm}$. The 1,1,3,3-tetramethoxypropane was used as an external standard and the level of lipid peroxidation was expressed as $\mu$ mol of malondialdehyde (MDA) per $2.5 \mathrm{mg}$ of tissue protein. Superoxide dismutase (SOD) activity in rat LVs was measured using the SOD Assay Kit-WST (Dojindo Molecular Technologies, Gaithersburg, MD, USA). Catalase activity in rat LVs was measured using a catalase assay kit (No. 707002) (Cayman Chemical, Ann Arbor, MI, USA). Glutathione peroxidase (GPx) activity in rat LVs was measured using a GPx assay kit (No. 03102) (Cayman Chemical).

\section{Carnitine Concentration in Rat LVs}

Carnitine concentrations were measured using the technique of Millington et al. ${ }^{17}$ In brief, LV tissue (10-15 mg) was homogenized with $1.5 \mathrm{ml}$ of Krebs-Henseleit solution. Following homogenization, $0.1 \mathrm{ml}$ of this homogenized solution, $0.05 \mathrm{ml}$ of the internal standard (Labeled carnitine standards SET B. Cambridge Isotope Laboratories, Andover, MA, USA) and $0.9 \mathrm{ml}$ of methanol were vortexed and centrifuged at $200 \mathrm{~Hz}$ for $10 \mathrm{~min}$. The resulting supernatant was dried under nitrogen. The residue was methylated by $5 \%$ hydrogen chloride in methanol and heated at $65^{\circ} \mathrm{C}$ for $15 \mathrm{~min}$. Methanol was then evaporated under a stream of nitrogen and the residue was reconstituted with $20 \mu 1$ of $50 \%$ glycerol in methanol containing $1 \%$ octylsulfate. A triple stage quadruple mass spectrometer, QUATTRO (Micromass, Manchester, UK), was used with an ion gun of cesium iodide as a secondary ion mass ionization mode. Predominant daughter ions of both labeled and unlabeled standards were $\mathrm{m} / \mathrm{z} 117$ for free carnitine, and, m/z 99 for acylcarnitines. 
Table 1 Effects of Chronic Hypoxia on Physical Characteristics of Juvenile Rats

\begin{tabular}{lcccc}
\hline \hline & Normoxia & Hypoxia & Normoxia $+\mathrm{O}_{2}$ & Hypoxia $+\mathrm{O}_{2}$ \\
\hline$n$ & 7 & 14 & 7 & 14 \\
$B W(\mathrm{~g})$ & $209 \pm 26$ & $114 \pm 24^{*}$ & $217 \pm 18$ & $122 \pm 23^{* *}$ \\
$R V W(\mathrm{mg})$ & $146 \pm 20$ & $138 \pm 37$ & $146 \pm 21$ & $151 \pm 34$ \\
$L V S W(\mathrm{mg})$ & $479 \pm 26$ & $295 \pm 86^{*}$ & $482 \pm 76$ & $333 \pm 62^{* *}$ \\
$H W / B W(\mathrm{mg} / \mathrm{g})$ & $3.01 \pm 0.27$ & $3.78 \pm 0.43^{*}$ & $2.88 \pm 0.21$ & $3.97 \pm 0.28^{* *}$ \\
$R V W / B W(\mathrm{mg} / \mathrm{g})$ & $0.70 \pm 0.06$ & $1.21 \pm 0.14^{*}$ & $0.67 \pm 0.07$ & $1.23 \pm 0.15^{* *}$ \\
$L V S W / B W(\mathrm{mg} / \mathrm{g})$ & $2.31 \pm 0.23$ & $2.57 \pm 0.35$ & $2.21 \pm 0.21$ & $2.74 \pm 0.20^{* *}$ \\
$R V W / L V S W$ & $0.30 \pm 0.03$ & $0.48 \pm 0.07 *$ & $0.31 \pm 0.04$ & $0.45 \pm 0.05^{* *}$ \\
\hline
\end{tabular}

Data are mean \pm standard deviation $(S D)(n=7-14)$.

${ }^{*} p<0.05$ vs normoxic animals. $* * p<0.05$ vs normoxic animals with hyperoxia.

$\mathrm{O}_{2}$, hyperoxia; $B W$, body weight; $R V W$, right ventricular free wall weight; LVSW, left ventricular free wall plus septum weight; $H W$, heart weight.

Table 2 Fatty Acid Composition (mol\%) of Total Fatty Acids in Left Ventricular Tissue of Rats Subjected to Hypoxia or Normoxia

\begin{tabular}{|c|c|c|c|c|c|c|c|c|c|c|}
\hline & \multicolumn{5}{|c|}{$L C A R(-)$} & \multicolumn{5}{|c|}{$\operatorname{LCAR}(+)$} \\
\hline & Normoxia & Hypoxia & $\begin{array}{c}\text { Normoxia } \\
\quad+\mathrm{O}_{2}\end{array}$ & $\begin{array}{c}\text { Hypoxia } \\
+\mathrm{O}_{2}\end{array}$ & $p$ value & Normoxia & Hyрохіа & $\begin{array}{c}\text { Normoxia } \\
+\mathrm{O}_{2}\end{array}$ & $\begin{array}{c}\text { Hypoxia } \\
+\mathrm{O}_{2}\end{array}$ & p value \\
\hline C16:0 & $12.7 \pm 2.4$ & $11.8 \pm 1.3$ & $12.1 \pm 2.0$ & $11.6 \pm 1.1$ & 0.69 & $10.2 \pm 0.4$ & $12.0 \pm 0.5^{*}$ & $11.5 \pm 1.5$ & $11.4 \pm 0.3$ & 0.028 \\
\hline C18:0 & $18.2 \pm 1.7$ & $16.7 \pm 0.5$ & $17.5 \pm 1.3$ & $16.1 \pm 0.9 *$ & 0.019 & $18.6 \pm 0.6$ & $16.0 \pm 0.1$ & $19.2 \pm 0.9$ & $17.8 \pm 0.7$ & 0.01 \\
\hline C18:1 & $7.2 \pm 0.5$ & $6.0 \pm 0.8 *$ & $7.4 \pm 0.6$ & $6.0 \pm 0.4 *$ & $<0.01$ & $6.20 \pm 0.4$ & $5.5 \pm 0.2 *$ & $6.7 \pm 0.3$ & $6.1 \pm 0.5$ & $<0.01$ \\
\hline$C 18: 2(n-6)$ & $22.8 \pm 3.8$ & $16.8 \pm 1.9 *$ & $23.7 \pm 3.2$ & $16.5 \pm 1.4 *$ & $<0.01$ & $17.0 \pm 1.1$ & $11.7 \pm 0.4 *$ & $20.5 \pm 5.2$ & $17.5 \pm 1.3$ & $<0.01$ \\
\hline$C 20: 4(n-6)$ & $20.0 \pm 1.5$ & $21.1 \pm 1.8$ & $20.3 \pm 3.0$ & $20.3 \pm 0.5$ & 0.7 & $24.4 \pm 1.1$ & $21.9 \pm 0.5$ & $24.1 \pm 2.5$ & $19.7 \pm 0.4$ & $<0.01$ \\
\hline$C 22: 5(n-3)$ & $1.3 \pm 0.3$ & $1.2 \pm 0.5$ & $0.7 \pm 0.4$ & $1.3 \pm 0.4$ & 0.054 & $2.1 \pm 0.2$ & $2.6 \pm 0.1$ & $1.5 \pm 0.7$ & $2.1 \pm 0.1$ & $<0.01$ \\
\hline$C 22: 6(n-3)$ & $16.8 \pm 4.5$ & $25.1 \pm 1.1 *$ & $17.3 \pm 3.6$ & $27.0 \pm 3.5 *$ & $<0.01$ & $19.8 \pm 0.4$ & $26.6 \pm 0.7 *$ & $15.0 \pm 2.1$ & $23.5 \pm 1.1 *$ & $<0.01$ \\
\hline$S F A$ & $31.5 \pm 3.5$ & $29.5 \pm 1.1$ & $30.2 \pm 2.7$ & $28.6 \pm 2.1$ & 0.209 & $29.9 \pm 0.8$ & $31.3 \pm 0.5$ & $31.5 \pm 2.0$ & $30.6 \pm 0.7$ & 0.13 \\
\hline$M U F A$ & $7.4 \pm 0.6$ & $6.1 \pm 0.8 *$ & $7.6 \pm 0.7$ & $6.1 \pm 0.5 *$ & $<0.01$ & $6.4 \pm 0.4$ & $5.6 \pm 0.2 *$ & $7.0 \pm 0.3$ & $6.3 \pm 0.2$ & $<0.01$ \\
\hline PUFA (n-3) & $18.3 \pm 4.7$ & $26.5 \pm 2.1 *$ & $18.2 \pm 3.8$ & $28.5 \pm 3.4 *$ & $<0.01$ & $22.3 \pm 0.5$ & $29.5 \pm 0.8 *$ & $16.9 \pm 2.6$ & $25.9 \pm 1.1 *$ & $<0.01$ \\
\hline$P U F A(n-6)$ & $42.8 \pm 2.6$ & $38.0 \pm 1.3 *$ & $44.0 \pm 2.4$ & $36.8 \pm 1.4^{*}$ & $<0.01$ & $41.4 \pm 0.7$ & $33.6 \pm 0.7 *$ & $44.6 \pm 4.1$ & $37.3 \pm 1.2 *$ & $<0.01$ \\
\hline
\end{tabular}

Values are mean \pm SD of 7 hearts per group.

Statistical significance of differences between the means was determined by 1-way ANOVA. *p<0.05 vs Normoxia.

LCAR, L-carnitine; SFA, saturated fatty acids; MUFA, mono-unsaturated fatty acids; PUFA, polyunsaturated fatty acids. Other abbreviation see in Table 1.

Fatty acids reaching at least $1 \%$ of the total shown only.

Table 3 Fatty Acid Composition (mol\%) of Phosphatidylethanolamine in Left Ventricular Tissue of Rats Subjected to Hypoxia or Normoxia

\begin{tabular}{|c|c|c|c|c|c|c|c|c|c|c|}
\hline & \multicolumn{5}{|c|}{$L C A R(-)$} & \multicolumn{5}{|c|}{$\operatorname{LCAR}(+)$} \\
\hline & Normoxia & Hурохіа & $\begin{array}{c}\text { Normoxia } \\
+\mathrm{O}_{2}\end{array}$ & $\begin{array}{c}\text { Hypoxia } \\
+\mathrm{O}_{2}\end{array}$ & $p$ value & Normoxia & Hyрохіа & $\begin{array}{c}\text { Normoxia } \\
+\mathrm{O}_{2}\end{array}$ & $\begin{array}{c}\text { Hypoxia } \\
+\mathrm{O}_{2}\end{array}$ & $p$ value \\
\hline C16:0 & $8.1 \pm 1.5$ & $9.3 \pm 0.8$ & $7.3 \pm 0.3$ & $9.8 \pm 1.8$ & 0.051 & $8.7 \pm 0.7$ & $8.9 \pm 0.7$ & $8.6 \pm 1.9$ & $10.1 \pm 1.9$ & 0.54 \\
\hline C18:0 & $21.0 \pm 3.5$ & $26.9 \pm 7.1$ & $23.4 \pm 7.4$ & $24.1 \pm 5.3$ & 0.6 & $21.9 \pm 1.4$ & $18.1 \pm 1.1$ & $29.3 \pm 4.9$ & $27.0 \pm 9.7$ & 0.053 \\
\hline C18:1 & $7.3 \pm 1.2$ & $4.4 \pm 2.1$ & $6.0 \pm 0.9$ & $4.4 \pm 1.1 *$ & 0.035 & $7.4 \pm 1.7$ & $5.8 \pm 0.6$ & $4.5 \pm 0.5^{*}$ & $4.7 \pm 1.4 *$ & 0.015 \\
\hline C18:2 (n-6) & $17.5 \pm 3.2$ & $8.7 \pm 6.5$ & $13.8 \pm 3.4$ & $8.5 \pm 4.0$ & 0.042 & $16.7 \pm 1.2$ & $11.0 \pm 2.2$ & $14.4 \pm 4.9$ & $7.2 \pm 3.8$ & 0.01 \\
\hline$C 20: 4(n-6)$ & $18.3 \pm 2.1$ & $17.0 \pm 1.6$ & $19.3 \pm 1.0$ & $17.2 \pm 1.3$ & 0.17 & $19.1 \pm 1.9$ & $15.4 \pm 0.8^{*}$ & $20.6 \pm 2.3$ & $16.0 \pm 0.6$ & $<0.01$ \\
\hline$C 22: 5(n-3)$ & $2.2 \pm 0.2$ & $1.4 \pm 0.5$ & $2.6 \pm 0.9$ & $1.2 \pm 0.4$ & 0.02 & $2.2 \pm 0.7$ & $2.0 \pm 0.3$ & $1.1 \pm 0.6$ & $2.1 \pm 0.4$ & 0.047 \\
\hline$C 22: 6(n-3)$ & $24.0 \pm 2.5$ & $31.3 \pm 1.8 *$ & $26.2 \pm 0.9$ & $33.5 \pm 3.3 *$ & $<0.01$ & $22.1 \pm 1.0$ & $37.2 \pm 1.7 *$ & $20.5 \pm 2.3$ & $34.8 \pm 1.5 *$ & $<0.01$ \\
\hline$S F A$ & $29.9 \pm 5.2$ & $36.8 \pm 7.5$ & $31.3 \pm 7.4$ & $34.6 \pm 6.9$ & 0.49 & $31.5 \pm 2.1$ & $27.8 \pm 1.0$ & $38.4 \pm 6.9$ & $34.6 \pm 5.8$ & 0.04 \\
\hline$M U F A$ & $7.7 \pm 1.2$ & $4.6 \pm 2.3$ & $6.3 \pm 1.0$ & $4.6 \pm 1.2$ & 0.04 & $7.8 \pm 1.9$ & $6.1 \pm 0.6$ & $4.6 \pm 0.6^{*}$ & $4.9 \pm 1.5 *$ & 0.75 \\
\hline PUFA (n-3) & $26.6 \pm 2.2$ & $32.9 \pm 1.5 *$ & $29.3 \pm 3.5$ & $35.1 \pm 3.5 *$ & $<0.01$ & $24.9 \pm 0.6$ & $39.7 \pm 2.0 *$ & $21.9 \pm 2.5$ & $37.4 \pm 1.7 *$ & $<0.01$ \\
\hline$P U F A(n-6)$ & $35.8 \pm 4.7$ & $25.7 \pm 6.0 *$ & $33.2 \pm 3.2$ & $25.7 \pm 3.4^{*}$ & 0.02 & $35.8 \pm 2.9$ & $27.9 \pm 1.8 *$ & $35.0 \pm 6.4$ & $23.2 \pm 3.6 *$ & $<0.02$ \\
\hline
\end{tabular}

Values are mean $\pm S D$ of 7 hearts per group.

Statistical significance of differences between the means was determined by 1-way ANOVA. *p<0.05 vs Normoxia.

Abbreviations see in Tables 1,2.

Fatty acids reaching at least $1 \%$ of the total shown only.

Statistical Analysis

Results are presented as the mean \pm the standard deviation. Statistical significance between more than 2 groups was tested using 1-way ANOVA followed by multiple comparisons with a Scheffé test. Student's t-test was used to compare mean values between 2 groups. Differences were considered to be statistically significant at a p-value $<0.05$.

\section{Results}

\section{Weight Parameters}

Hypoxia significantly decreased the body weight of the rats, and increased the ratio of heart weight to body weight as compared with the age-matched normoxic controls (Table 1). The latter effect was mainly because of right ventricular hypertrophy derived from enhanced pulmonary 
Table 4 Fatty Acid Composition (mol\%) of Phosphatidylcholine in Left Ventricular Tissue of Rats Subjected to Hypoxia or Normoxia

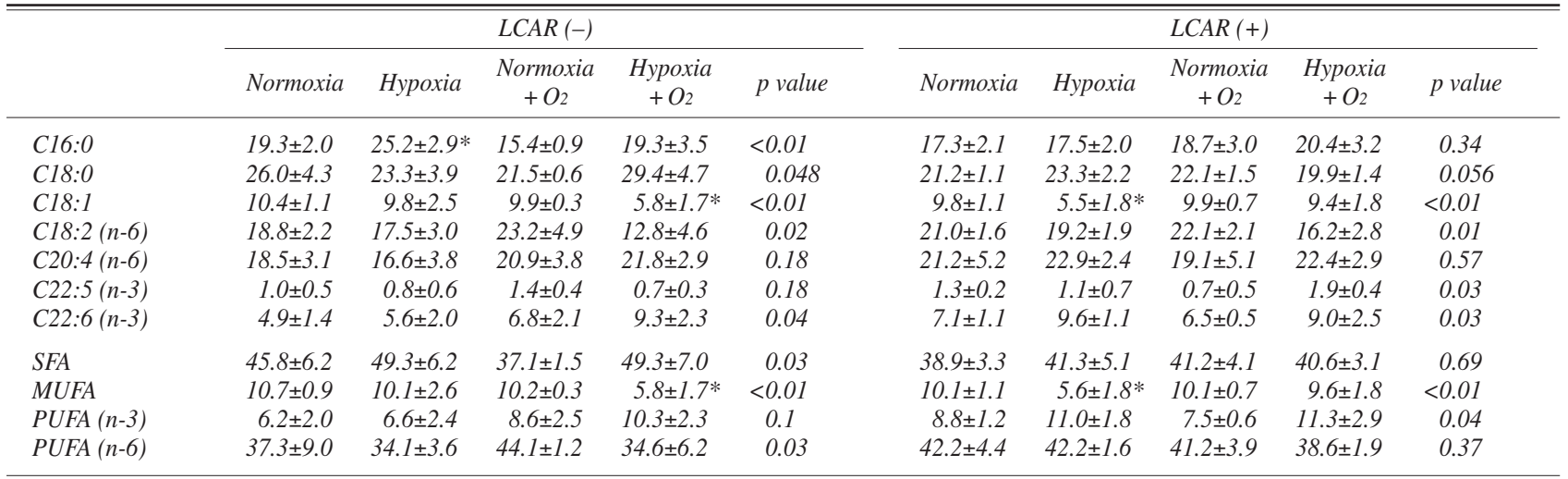

Values are mean \pm SD of 7 hearts per group.

Statistical significance of differences between the means was determined by 1-way ANOVA. *p<0.05 vs Normoxia.

Abbreviations see in Tables 1,2.

Fatty acids reaching at least $1 \%$ of the total shown only.

\section{n-3 PUFA}

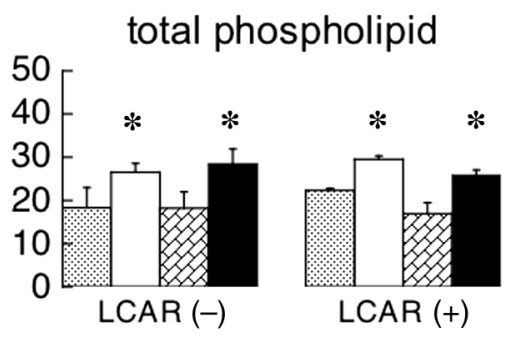

$\mathrm{PE}$

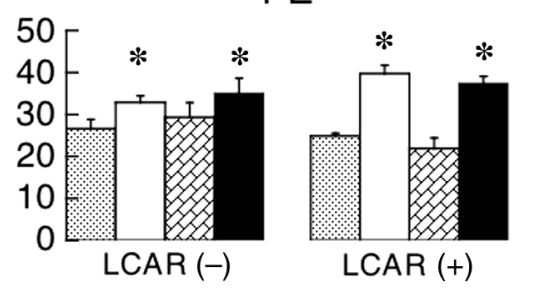

$\mathrm{PC}$

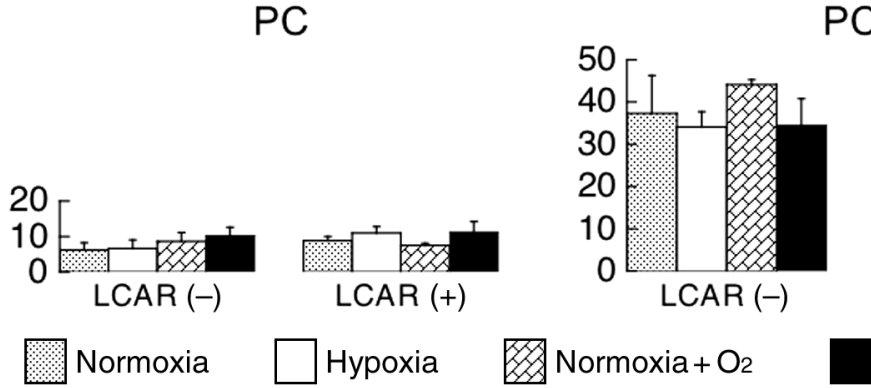

\section{n-6 PUFA}

total phospholipid
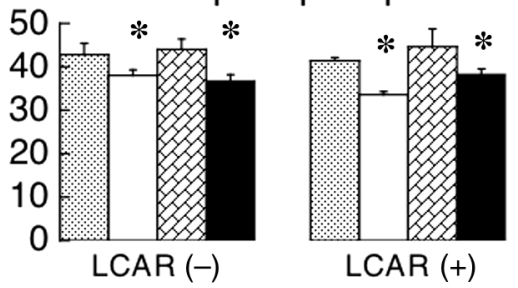

$\mathrm{PE}$

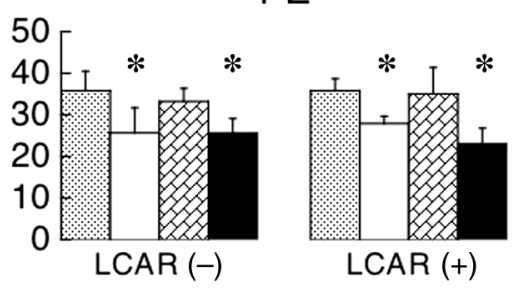

PC

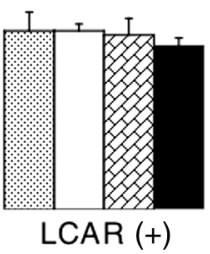

Hypoxia $+\mathrm{O}_{2}$
Fig 1. Main fatty acid classes of total phospholipid, phosphatidylethanolamine (PE) and phosphatidylcholines (PC) in the left ventricles of chronically hypoxic rats. Values are expressed as $\% \mathrm{~mol} \pm$ standard deviation. In the total phospholipids and PE, the proportions of n-6 PUFA were decreased and those of n-3 PUFA were increased by hypoxia. This fatty acid profile was unchanged by the addition of 1-carnitine (LCAR) and 12-h exposure of hyperoxia. The proportion of $n-3$ PUFA in PC was low and there was no remarkable change in either n-3 or n-6 PUFA level. $* \mathrm{p}<0.05$ vs Normoxia, $\mathrm{n}=7$ for each group. PUFA, polyunsaturated fatty acid. vascular resistance under hypoxic conditions. Thus, the ratio of right ventricular free wall weight to body weight showed a marked increase in hypoxic animals $(170 \%$ of the values for the normoxic rats). However, the ratio of LV free wall plus septum weight to body weight in the "Hypoxia" group did not change significantly compared with the "Normoxia" group.

\section{Total Fatty Acids and Phospholipid Analysis}

The fatty acid composition of total and individual phospholipid species is documented in Tables 2-4. Fig 1 illus- trates the effect of hypoxia on the distribution of the main fatty acids classes in the total and individual phospholipids.

Concentrations of total fatty acids in the LV myocardium in the "Hypoxia" and "Normoxia" groups are summarized in Table 2 and Fig 1. Saturated fatty acids (SFA) accounted for approximately $32 \%$ of the total fatty acids in normoxic ventricles, with n-6 polyunsaturated fatty acids (PUFA) and n-3 PUFA accounting for approximately $43 \%$ and $18 \%$ of total fatty acids, respectively. Hypoxia decreased the proportion of n-6 PUFA, particularly linoleic acid (C18:2), which was accompanied by a proportional increase in $n-3$ 


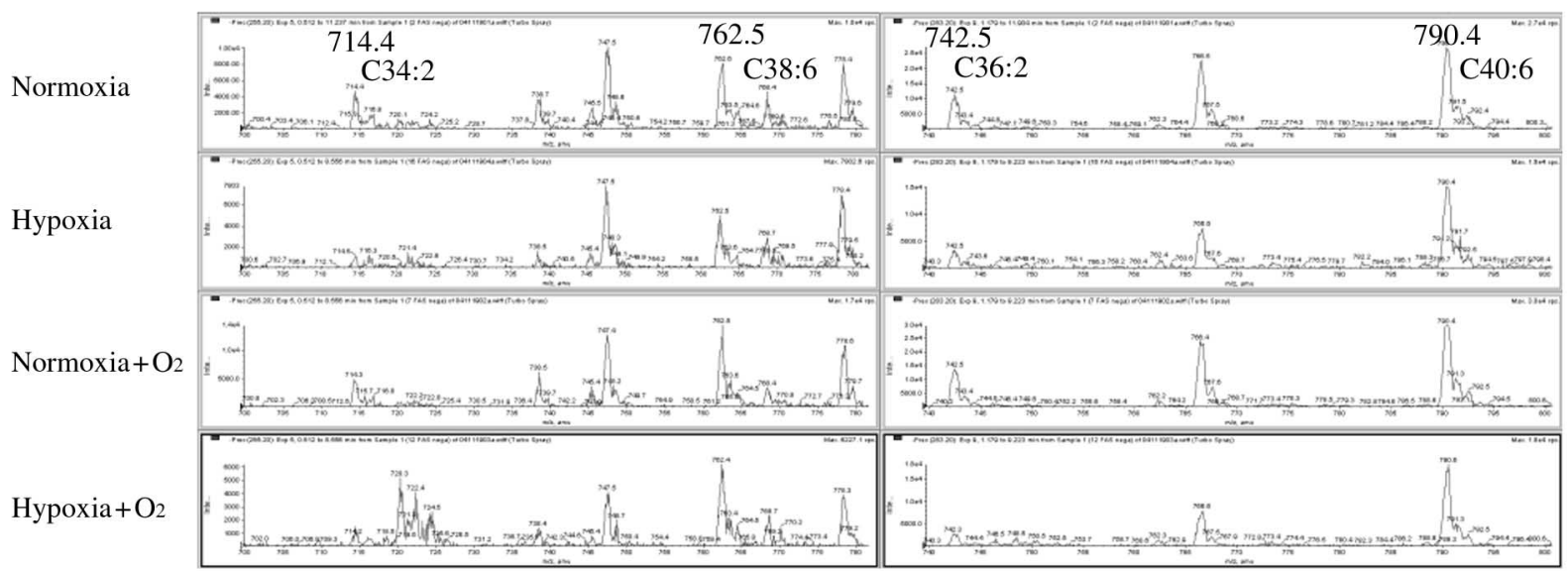

Fig 2. Typical phosphatidylethanolamine profile analyzed by Cap-LC/ESI mass spectrometry. The main fatty acid constituents were palmitic acid (C16:0), stearic acid (C18:0), linoleic acid (C18:2), arachidonic acid (C20:4) and docosahexaenoic acid (C22:6). Because the change in $\mathrm{C} 20: 4$ was not remarkable, the conceivable combinations were $\mathrm{C} 34: 2$ (C16:0-C18:2), C38:6 (C16:0-C22:6), C36:2 (C18:0-C18:2), and C40:6 (C18:0-C22:6). Molecular species of mass/charge $(\mathrm{m} / \mathrm{z})$ of $\mathrm{C} 34: 2$ was 714.4 , that of $\mathrm{C} 38: 6$ was 762.5 , that of $\mathrm{C} 36: 2$ was 742.5 , and that of $\mathrm{C} 40: 6$ (C 18:0C22:6) was 790.4. LC/ESI, liquid choromatography-electrospray ionization.

PUFA, particularly docosahexaenoic acid (C22:6, DHA). This fatty acid profile was unchanged with the addition of LCAR (Fig 1).

The distribution of fatty acids in PE is presented in Table 3 and Fig 1. SFA accounted for approximately 30\% of PE fatty acid, n-6 PUFA for approximately $36 \%$, and n-3 PUFA for approximately $27 \%$ in normoxic ventricles. Similar to what was observed for total fatty acids, hypoxia decreased n-6 PUFA and increased n-3 PUFA. The effect was also pronounced with the addition of LCAR (Fig 1).

The fatty acid composition of phosphatidylcholine (PC) in the LV is shown in Table 4 and Fig 1. SFA accounted for approximately $46 \%$ of PC fatty acid, n-6 PUFA for approximately $37 \%$, and n-3 PUFA for approximately $6 \%$ in normoxic ventricles. The proportion of n-3 PUFA in PC was low and there was no remarkable change in either $n-6$ or n-3 PUFA (Fig 1).

ESI-MS elucidated the fatty acid composition of the total fatty acids and phospholipids. In general, the fatty acid forms in the sn1 position of phospholipid were SFA and fatty acids at the sn 2 position were PUFA. The main fatty acids in phospholipids were palmitic acid (C16:0), stearic acid (C18:0), linoleic acid (C18:2), arachidonic acid (C20:4) and DHA (C22:6). The change in C20:4 in the "Hypoxia" group was not remarkable. The conceivable combinations were C16:0-C18:2 (C34:2), C16:0-C22:6 (C38:6), C18:0C18:2 (C36:2), and C18:0-C22:6 (C40:6). The structure of $\mathrm{PE}$ is shown in Fig 2. C34:2 (C16:0-C18:2) and C36:2 (C18:0-C18:2) levels were lower in the "Hypoxia" group compared with the "Normoxia" group. In contrast, C38:6 (C16:0-C22:6) and C40:6 (C18:0-C22:6) were higher in the "Hypoxia" group compared with the levels in the "Normoxia" group.

\section{TBARs Measurement}

The MDA levels in the "Normoxia", "Hypoxia", "Normo$\mathrm{xia}+\mathrm{O} 2$ " and "Hypoxia $+\mathrm{O}_{2}$ " groups were $1.9 \pm 0.7,1.4 \pm 0.7$, $2.2 \pm 0.6$, and $6.3 \pm 2.3 \mu \mathrm{mol} / 2.5 \mathrm{-mg}$ protein, respectively. MDA levels were not increased in the "Hypoxia" group compared with the levels in the "Normoxia" group $(\mathrm{p}=0.75)$, but were elevated in the "Hypoxia $+\mathrm{O}_{2}$ " group compared
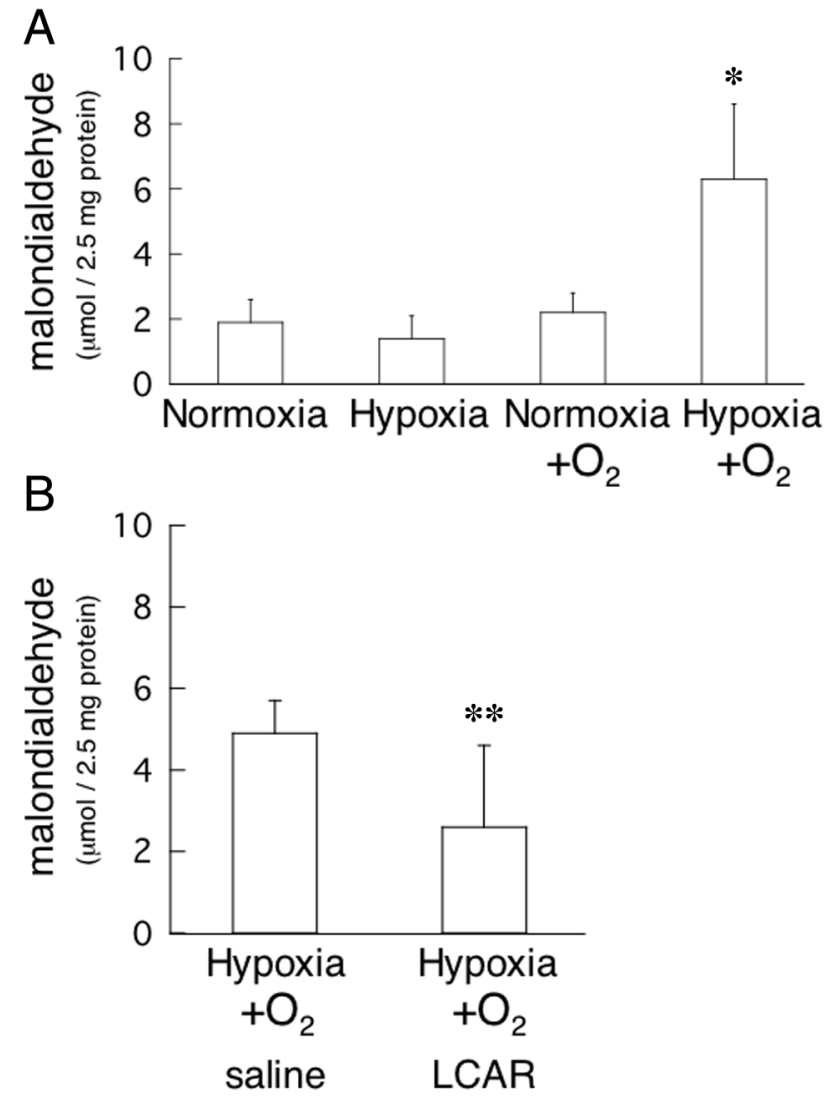

Fig 3. Lipid peroxidation measured as malondialdehyde (MDA) content after 2 weeks of hypoxia (10\% oxygen) and $12 \mathrm{~h}$ hyperoxia (100\% oxygen). Values are expressed as $\mu \mathrm{mol} / 2.5-\mathrm{mg}$ protein. (A) MDA levels: Normoxia, Hypoxia, Normoxia $+\mathrm{O}_{2}$, and Hypoxia $+\mathrm{O}_{2}$. $* \mathrm{p}<0.05$ for Hypoxia $+\mathrm{O}_{2}$ vs Normoxia $+\mathrm{O}_{2}$. All values are represented as mean \pm standard deviation, $n=7$ for each group. (B) Effect of 2-week 1-carnitine (LCAR) addition in the Hypoxia $+\mathrm{O}_{2}$ group. LCAR $(200 \mathrm{mg} / \mathrm{kg}$ ) was administered by intraperitoneal injection (ip) daily for 2 weeks. Saline was administered by ip daily for 2 weeks to the control group. $* * p<0.05$ for Hypoxia $+\mathrm{O}_{2}+$ saline vs Hypoxia + $\mathrm{O}_{2}+\mathrm{LCAR}, \mathrm{n}=5$ for both groups. 
Table 5 Carnitine Concentration (nmol/g Dry Weight) in Left Ventricular Tissue of Rats Subjected to Hypoxia or Normoxia

\begin{tabular}{lcccccc}
\hline \hline & Normoxia & Normoxia + LCAR & p value & Hypoxia & Hypoxia + LCAR & p value \\
\hline Free & $9.3 \pm 3.3$ & $8.4 \pm 1.3$ & 0.72 & $6.6 \pm 0.9$ & $6.1 \pm 3.1$ & 0.38 \\
C2 & $2.5 \pm 0.7$ & $3.6 \pm 0.4$ & 0.011 & $2.4 \pm 0.9$ & $3.5 \pm 0.6$ & 0.03 \\
C3 & $0.056 \pm 0.014$ & $0.136 \pm 0.065$ & 0.023 & $0.073 \pm 0.010$ & $0.089 \pm 0.026$ & 0.12 \\
C4 & $0.010 \pm 0.007$ & $0.064 \pm 0.054$ & 0.49 & $0.022 \pm 0.011$ & $0.058 \pm 0.033$ & 0.03 \\
C5 & $0.006 \pm 0.003$ & $0.014 \pm 0.004$ & 0.013 & $0.016 \pm 0.004$ & $0.014 \pm 0.004$ & 0.74 \\
Total of C1-C5 & $11.9 \pm 3.7$ & $12.2 \pm 1.6$ & 0.44 & $9.2 \pm 1.0$ & $9.8 \pm 3.2$ & 0.36 \\
\hline
\end{tabular}

Values are mean \pm SD of 6 hearts per group.

C2, acetylcarnitine; C3, propionylcarnitine; C4, C4-acylcarnitine; C5, C5-acylcarnitine. Other abbreviation see in Table 2.

with the "Normoxia $+\mathrm{O} 2$ " group $(\mathrm{p}<0.01)(\mathrm{Fig} 3 \mathrm{~A})$. The administration of LCAR to the "Hypoxia $+\mathrm{O}_{2}$ " group almost completely suppressed the increased cardiac lipid peroxidation that was observed in only the "Hypoxia $+\mathrm{O}_{2}+$ saline" group $(2.6 \pm 2.0 \mu \mathrm{mol} / 2.5-\mathrm{mg}$ protein vs $4.9 \pm 0.8 \mu \mathrm{mol} /$ 2.5-mg protein, $\mathrm{p}<0.05)$ (Fig 3B).

\section{Level of Antioxidants}

Tissue SOD activity in the "Hypoxia" group was significantly decreased compared with the activity in the "Normoxia" group $(89.7 \pm 2.6 \%$ vs $94.9 \pm 1.4 \%, \mathrm{p}<0.01)$. Following the administration of LCAR, SOD activity did not change ("Hypoxia+LCAR" $88.8 \pm 2.6 \%$ vs "Normoxia +LCAR" $94.8 \pm 3.4 \%, \mathrm{p}<0.01)$. Catalase activity and GPx activity were not different between the "Normoxia" and "Hypoxia" groups (Catalase: "Normoxia" $5.6 \pm 1.1 \mathrm{nmol} / \mathrm{kg}$ dry weight $/ \mathrm{min}$; "Hypoxia" $6.4 \pm 1.5 \mathrm{nmol} / \mathrm{kg}$ dry weight $/ \mathrm{min}, \mathrm{p}=0.053$, GPx: "Normoxia" $0.57 \pm 0.1 \mu \mathrm{mol} \cdot \mathrm{min}^{-1} \cdot \mathrm{mg}$ dry weight ${ }^{-1}$, "Hypoxia" $0.45 \pm 0.1 \mu \mathrm{mol} \cdot \mathrm{min}^{-1} \cdot \mathrm{mg}$ dry weight ${ }^{-1}, \mathrm{p}=0.09$ ). Following the administration of LCAR, catalase activity and GPx activity did not change (Catalase: "Normoxia +LCAR" $5.3 \pm 1.3 \mathrm{nmol} \cdot \mathrm{kg}$ dry weight ${ }^{-1} \cdot \mathrm{min}^{-1}$, and "Hypoxia + LCAR" $5.4 \pm 1.1 \mathrm{nmol} \cdot \mathrm{kg}$ dry weight ${ }^{-1} \cdot \mathrm{min}^{-1}$, GPx: "Normoxia + LCAR" $0.43 \pm 0.1 \mu \mathrm{mol} \cdot \mathrm{min}^{-1} \cdot \mathrm{mg}^{-1}$ dry weight ${ }^{-1}$, and "Hypoxia +LCAR" $0.46 \pm 0.1 \mu \mathrm{mol} \cdot \mathrm{min}^{-1} \cdot \mathrm{mg}$ dry weight ${ }^{-1}$.

\section{Cardiac Carnitine Concentrations}

Administration of LCAR significantly increased the acetylcarnitine $(\mathrm{C} 2)$ concentration in both the "Normoxia" and "Hypoxia" groups (Table 5). The free carnitine concentration in the heart tissue did not differ between the "Normoxia" and "Hypoxia" groups. The amount of free carnitine, acetylcarnitine (C2), propionylcarnitine (C3), C4-, and C5acylcarnitine was also not different between the "Normoxia" and "Hypoxia" groups4

\section{Discussion}

We show that exposure of young rats to 2 weeks of hypoxia caused changes in the fatty acid composition and scavenger enzyme activity in the heart. Of importance is the age of the rats. One reason for using 4-week-old rats is the difference in mortality rates we observed with age. In preliminary studies, we exposed male rats immediately at birth to hypoxia for 2 weeks, but the survival rate was quite low, whereas the mortality rate is under $5 \%$ for the 4 -weekold rats. The impact of nutrition on lipid metabolism is also important because fatty acid composition is greatly influenced by meal composition. 18 The fatty acid composition did not change in the hypoxic rat heart immediately after birth (SFA: "Normoxia" 48.5 $\pm 2.9 \%$ mol, "Hypoxia" 47.0 2.3 , p=
0.85; MFA: "Normoxia" 4.3 \pm 0.5 , "Hypoxia" $5.8 \pm 0.5$, p $<$

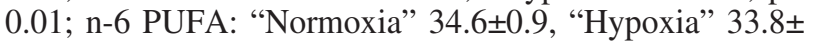
1.4, $\mathrm{p}=0.12$; $\mathrm{n}-3$ PUFA: "Normoxia" $12.6 \pm 1.7$, "Hypoxia" $13.4 \pm 2.1, \mathrm{p}=0.77)$. Mammals must obtain fatty acids with more than 2 double bonds (=PUFA) from the diet because they cannot synthesize them. Rats before weaning get PUFA from the dam's milk in which the proportion of PUFA in total fat is less than $20 \%$. After weaning, rats obtain PUFA from chow containing more than $60 \%$ of PUFA in the total fat content. From the clinical point of view, this dramatic change in the dietary PUFA supply influences the myocardial fatty acid composition and may be associated with myocardial injury by lipid peroxidation. Because this external effect of a different diet will complicate the consideration of myocardial fatty acid composition, we avoided the nutritional shift from breastfeeding to standard chow during this study. The fatty acid composition in the heart gradually changes during growth; the composition of n-3 PUFA decreases and that of n-6 PUFA increases after weaning 18 Based on those reports, we selected the starting point of hypoxic exposure as 4 weeks of age, just after weaning.

Two-week-hypoxia decreases cardiac n-6 PUFA content in the young rat, which was mainly attributable to a decrease in C18:2. There was also a concomitant increase in n-3 PUFA, which was mainly related to C22:6 in total lipids and PE. This finding is similar to previous reports of the fatty acid composition of cardiac phospholipids in membrane lipids in adolescents animals exposed to high doses of catecholamines ${ }^{19}$ aortic constriction ${ }^{20}$ or intermittent high altitude hypoxia21 Although exposure to chronic hypoxia is initially associated with an increase in adrenergic activity ${ }^{22,23}$ adrenoceptor blockade does not attenuate the changes in the fatty acid composition of phospholipids. ${ }^{20}$ In our preliminary study, the fatty acid composition of hypoxic rat hearts followed by 2 weeks ofnormoxia was not remarkably different in the comparison with that of normoxic rat hearts (data not shown). We believe from these results that the change in fatty acid composition after 2 weeks of hypoxic exposure is reversible by re-establishing a normoxic environment. It has been reported that the proportion of $n-3$ PUFA in rat heart PE is high, while the proportion in PC is low! ${ }^{18}$ However, the different roles of these 2 major phospholipids in cell membranes is not well known.

Recent studies suggest that the contribution of fatty acids to energy metabolism in the LV is unaffected by chronic hypoxic acclimatization. Adrogue et al reported chronic hypobaric hypoxia $\left(45 \mathrm{kPA}-11 \%\right.$ ambient $\mathrm{O}_{2}$, for a period of 4,10 , and 12 weeks) induced changes in metabolic gene expression, suggesting a downregulation of fatty acid metabolism and an increase in glucose metabolism in the 
right ventricle, while LV metabolic gene expression suggested a restoration of fatty acid metabolism? $?^{4}$ Thus, fatty acid metabolism may not have altered in our model of invivo hypoxia. When we consider the pathology of cyanotic $\mathrm{CHD}$, we enumerate 2 factors. One is the influence of the pressure- and volume-loads, and the second is the influence of hypoxia on the heart. We believe our experimental model allows investigation of the direct effects of hypoxia on LV fatty acid composition; however, as the LVSW/BW (LVSW: LV free wall plus septum weight, BW; body weight) is not altered by hypoxia, the experimental model is unable to assess the contribution of alterations in pressure- and/or volume load.

We also observed whether 2 weeks of hypoxia caused a decrease in scavenger enzyme activity in young rat hearts, compared with normoxic hearts. Rapid scavenging of superoxide $\left(\mathrm{O}_{2}-\right)$ radicals by $\mathrm{SOD}$, catalase and GPx activity prevents these extremely reactive products from attacking other molecules in the cell. As a result, decreased scavenging enzyme activity caused by ischemia or chronic hypoxia may render cells more susceptible to re-oxygenation injury. In the present study, hypoxia decreased myocardial SOD activity, but no change was observed in the tissue catalase or GPx activity. A decrease of SOD by hypoxia conforms to observations in other studies?,25

Our data also demonstrate that lipid peroxidation in the heart is only accelerated with hyperoxia after exposure to chronic hypoxia. Barrington et al have shown that free radicals are unlikely to be a major cause of mechanical dysfunction in reperfused hearts, because no increase in lipid peroxidation occurred during the reperfusion period 26 The failure of ischemia-reperfusion to increase lipid peroxidation is consistent with the absence of MDA increase during ischemia-reperfusion ${ }^{27} \mathrm{The}^{\mathrm{O}} 2$ - radical has the potential to react with any molecule, and it may lead to lipid peroxidation with the formation of a diene bond. This process can be propagated within the cell membrane until arrested by other scavenger systems. Hypoxia per se does not raise the myocardial lipid peroxidation level, as shown in both our and other studies ${ }^{28,29}$ Ihnken et al reported that hypoxia increases cardiac susceptibility to oxidant damage by reducing antioxidants, and re-oxygenation further depletes the antioxidant level and accelerates lipid peroxidation 28 Lipid peroxidation occurs by oxidation of PUFA in microsomal phospholipids. Our results suggest that increased lipid peroxidation by hyperoxia in hypoxic rat hearts may be related to an increase in PUFA content, which has more double bonds and is therefore more susceptible to lipid peroxidation. In chronically hypoxic rats, a shift in favor of n-3 PUFA and a decrease in the level of antioxidants might be necessary to accelerate lipid peroxidation following acute exposure to oxygen. Although MDA was increased in the hypoxia $+\mathrm{O}_{2}$ group ( $\mathrm{Fig} 3 \mathrm{~A}$ ), there were no differences in the fatty acid composition between the hypoxia and hypo$\mathrm{xia}+\mathrm{O}_{2}$ groups (Fig 1), possibly because of lipid peroxidation occurring in specific subcellular compartments (eg, mitochondria) ${ }^{30}$ and thus having been underrepresented in the extracts of LV tissue used in this study. Alternatively, it may be because of differences in the rate of formation of n-3 PUFAs (from n-6 PUFAs) and the rate of formation of MDA.

Several reports have suggested that carnitine derivatives act as free radical scavengers?-11 In support of this, we showed that carnitine effectively scavenges superoxide anions, resulting in inhibition of lipid peroxidation. Our study results revealed that daily addition of LCAR increased the acetylcarnitine concentration in rat hearts. Recent reports show that acetylcarnitine ${ }^{9}$ and propionyl carnitine $e^{10,11}$ possess potent antioxidant activity. The free radical scavenging activity of acylcarnitine has been shown to be dosedependent?-11,31 The increase in tissue acetyl carnitine concentration induced by LCAR administration may effectively prevent lipid peroxidation. Although LCAR reduced the levels of lipid hydroperoxide in the heart after oxidative stress, the antioxidant effects of LCAR and its relationship with the composition of membrane lipid bilayers remain to be clarified. In the present study, phospholipid composition remained unchanged in the presence of LCAR in both the "Normoxia" and "Hypoxia" groups. Moreover, LCAR did not affect SOD activity, catalase concentration or GPx activity. Reznick et al have shown that LCAR suppresses hydroxyl radical production in the Fenton reaction! ${ }^{10}$ Furthermore, LCAR inhibited xanthine oxidase activity, thus acting as a free radical scavenger and stabilizer of cell membranes. $^{32}$

\section{Experimental Limitations}

Potential limitations of this study are as follows.

(1) Children with cyanotic congenital heart defects are generally in a hypoxic condition after birth and are not exposed to normoxia until repair of their congenital heart abnormalities. Thus, our animal model did not exactly mimic the clinical setting because the rats were 4 weeks old before exposure to hypoxia. However, as discussed, it is hard to use neonatal hypoxic models because the mortality rate is high. We selected a hypoxic period of 2 weeks, as this time period has been characterized and well established in adult rat hearts! 12 Specifically, the previous report indicates that various parameters affected by hypoxia reach a steady-state level over this time period $!^{2}$ In our preliminary study, 2 days of inhalation hypoxia did not influence cardiac fatty acid composition (data not shown). In the present study, we have used this model of hypoxia to study alterations in cardiac fatty acid composition in the juvenile rat heart.

(2) Body weight of the hypoxic model was significantly different from that of the normoxic model, which is a similar result to that of another report! ${ }^{3}$ Hypoxia is associated with an increase in adrenergic activity ${ }_{20,21}$ which may be responsible for the reduction in body weight.

In conclusion, the present study shows that an increase in n-3 PUFA and a decrease in antioxidants occurs in young rat hearts under chronic hypoxia, which may augment lipid peroxidation following acute exposure to oxygen. LCAR administration increases the acetylcarnitine concentration in hypoxic heart tissue and suppresses the increase in lipid peroxidation following re-oxygenation, despite the lack of an effect on the fatty acid composition and antioxidant levels.

\section{Acknowledgments}

We thank Dr Gary D. Lopaschuk at the University of Alberta for his invaluable suggestions.

\section{References}

1. Najm HK, Wallen WJ, Belanger MP, Williams WG, Coles JG, Van Arsdell GS, et al. Does the degree of cyanosis affect myocardial adenosine triphosphate levels and function in children undergoing surgical procedures for congenital heart disease? J Thorac Cardiovasc Surg 2000; 119: 515-524. 
2. del Nido PJ, Mickle DA, Wilson GJ, Benson LN, Coles JG, Trusler GA, et al. Evidence of myocardial free radical injury during elective repair of tetralogy of Fallot. Circulation 1987; 76: V-174-V-179.

3. Hu J, Sun P, Ruan X, Chao A, Lin Y, Li XY, et al. Mechanism of myocardial microvessel formation in cyanotic congenital heart disease. Circ J 2005; 69: 1089-1093.

4. Kajimoto H, Nakazawa M, Murasaki K, Mori Y, Tanoue K, Kasanuki $\mathrm{H}$, et al. Increased thrombogenesity in patients with cyanotic congenital heart disease. Circ J 2007; 71: 948-953.

5. Allen BS, Rahman S, Ilbawi MN, Kronon M, Bolling KS, Halldorsson $\mathrm{AO}$, et al. Detrimental effects of cardiopulmonary bypass in cyanotic infants: Preventing the reoxygenation injury. Ann Thorac Surg 1997; 64: $1381-1387$; discussion 1387-1388.

6. Ozer MK, Parlakpinar H, Cigremis Y, Ucar M, Vardi N, Acet A. Ischemia-reperfusion leads to depletion of glutathione content and augmentation of malondialdehyde production in the rat heart from overproduction of oxidants: Can caffeic acid phenethyl ester (CAPE) protect the heart? Mol Cell Biochem 2005; 273: 169-175.

7. Teoh KH, Mickle DA, Weisel RD, Li RK, Tumiati LC, Coles JG, et al. Effect of oxygen tension and cardiovascular operations on the myocardial antioxidant enzyme activities in patients with tetralogy of Fallot and aorta-coronary bypass. J Thorac Cardiovasc Surg 1992; 104: $159-164$

8. Ihnken K, Morita K, Buckberg GD. Delayed cardioplegic reoxygenation reduces reoxygenation injury in cyanotic immature hearts. Ann Thorac Surg 1998; 66: 177 - 182.

9. Yasui F, Matsugo S, Ishibashi M, Kajita T, Ezashi Y, Oomura Y, et al. Effects of chronic acetyl-L-carnitine treatment on brain lipid hydroperoxide level and passive avoidance learning in senescenceaccelerated mice. Neurosci Lett 2002; 334: 177-180.

10. Reznick AZ, Kagan VE, Ramsey R, Tsuchiya M, Khwaja S, Serbinova EA, et al. Antiradical effects in L-propionyl carnitine protection of the heart against ischemia-reperfusion injury: The possible role of iron chelation. Arch Biochem Biophys 1992; 296: 394-401.

11. Bertelli A, Conte A, Ronca G, Segnini D, Yu G. Protective effect of propionyl carnitine against peroxidative damage to arterial endothelium membranes. Int J Tissue React 1991; 13: 41-43.

12. Sharma S, Taegtmeyer H, Adrogue J, Razeghi P, Sen S, Ngumbela K, et al. Dynamic changes of gene expression in hypoxia-induced right ventricular hypertrophy. Am J Physiol Heart Circ Physiol 2004; 286: H1185-H1192.

13. Corno AF, Milano G, Morel S, Tozzi P, Genton CY, Samaja M, et al. Hypoxia: Unique myocardial morphology? J Thorac Cardiovasc Surg 2004; 127: 1301-1308.

14. Bligh EG, Dyer WJ. A rapid method of total lipid extraction and purification. Can J Biochem Physiol 1959; 37: 911-917.

15. Ozawa A, Jinpo H, Takahashi H, Fujita T, Hirai A, Terano T, et al. Determination of higher fatty acids in phospholipid subfractions of human platelets using thin-layer chromatography and gas chromatography. Bunseki Kagaku 1985; 34: 707 (in Japanese).

16. Ando K, Beppu M, Kikugawa K. Evidence for accumulation of lipid hydroperoxides during the aging of human red blood cells in the circulation. Biol Pharm Bull 1995; 18: 659-663.

17. Millington DS, Terada N, Chace DH, Chen YT, Ding JH, Kodo N, et al. The role of tandem mass spectrometry in the diagnosis of fatty acid oxidation disorders. Prog Clin Biol Res 1992; 375: 339-354.

18. Gudmundsdottir A, Gudbjarnason S. Neonatal changes in fatty acid profile of phospholipids in rat heart muscle. Biochim Biophys Acta 1983; 752: 284-290.

19. Benediktsdottir VE, Gudbjarnason S. Reversible alterations in fatty acid composition of heart muscle membrane phospholipids induced by epinephrine in rats fed different fats. J Lipid Res 1988; 29: 765 772 .

20. O'Rourke B, Reibel DK. Effects of adrenoceptor blockade on cardiac hypertrophy and myocardial phospholipids. Proc Soc Exp Biol Med 1992; 200: $95-100$.

21. Jezkova J, Novakova O, Kolar F, Tvrzicka E, Neckar J, Novak F. Chronic hypoxia alters fatty acid composition of phospholipids in right and left ventricular myocardium. Mol Cell Biochem 2002; 232: $49-56$.

22. Mojet MH, Mills E, Duchen MR. Hypoxia-induced catecholamine secretion in isolated newborn rat adrenal chromaffin cells is mimicked by inhibition of mitochondrial respiration. J Physiol 1997; 504: 175 189.

23. Johnson TS, Young JB, Landsberg L. Sympathoadrenal responses to acute and chronic hypoxia in the rat. J Clin Invest 1983; 71: $1263-$ 1272 .

24. Adrogue JV, Sharma S, Ngumbela K, Essop MF, Taegtmeyer H. Acclimatization to chronic hypobaric hypoxia is associated with a differential transcriptional profile between the right and left ventricle. Mol Cell Biochem 2005; 278: $71-78$.

25. Kawaguchi H, Shoki M, Iizuka K, Sano H, Sakata Y, Yasuda H. Phospholipid metabolism and prostacyclin synthesis in hypoxic myocytes. Biochim Biophys Acta 1991; 1094: 161-167.

26. Barrington PL, Lai E, McCay PB. Lack of myocardial lipid peroxidation during acute reperfusion injury in perfused guinea pig hearts. Cardiovasc Res 1993; 27: 1339-1345.

27. Liedtke AJ, Mahar CQ, Ytrehus K, Mjos OD. Estimates of freeradical production in rat and swine hearts: Method and application of measuring malondialdehyde levels in fresh and frozen myocardium. Basic Res Cardiol 1984; 79: 513-518.

28. Gauduel Y, Duvelleroy MA. Role of oxygen radicals in cardiac injury due to reoxygenation. J Mol Cell Cardiol 1984; 16: 459-470.

29. Ihnken K, Morita K, Buckberg GD, Matheis G, Sherman MP, Allen BS, et al. Studies of hypoxemic/reoxygenation injury: Without aortic clamping. II: Evidence for reoxygenation damage. J Thorac Cardiovasc Surg 1995; 110: $1171-1181$

30. Nojiri H, Shimizu T, Funakoshi M, Yamaguchi O, Zhou H, Kawakami $\mathrm{S}$, et al. Oxidative stress causes heart failure with impaired mitochondrial respiration. J Biol Chem 2006; 281: 33789-33801.

31. Vanella A, Russo A, Acquaviva R, Campisi A, Di Giacomo C, Sorrenti V, et al. L-propionyl-carnitine as superoxide scavenger, antioxidant, and DNA cleavage protector. Cell Biol Toxicol 2000; 16: 99-104.

32. Di Giacomo C, Latteri F, Fichera C, Sorrenti V, Campisi A, Castorina $\mathrm{C}$, et al. Effect of acetyl-L-carnitine on lipid peroxidationand xanthine oxidase activity in rat skeltal muscle. Neurochem Res 1993; 18: $1157-1162$. 